\title{
Future Roles of Lapatinib in ErbB2-Positive Breast Cancer: Adjuvant and Neoadjuvant Trials
}

\author{
Serban-Dan Costa $^{\mathrm{a}} \quad$ Christian Jackisch $^{\mathrm{b}} \quad$ Christoph Thomssen $^{\mathrm{c}}$ \\ a Universitätsfrauenklinik Magdeburg, \\ ${ }^{\mathrm{b}}$ Department of Obstetrics and Gynecology and Breast Cancer Center, Klinikum Offenbach, \\ c Universitätsklinik und Poliklinik für Gynäkologie, Halle/Saale, Germany
}

\section{Key Words}

Lapatinib - ErbB2-positive breast cancer .

Adjuvant · Neoadjuvant - ALTTO study · TEACH study

\section{Summary}

Lapatinib is potentially an ideal therapy for the adjuvant and neoadjuvant treatment of women with breast cancer due to its convenience of use (oral, once-daily administration) and because it has shown activity in the first-line and refractory metastatic settings. Furthermore, the dual tyrosine kinase inhibitor appears to have a low incidence of cardiotoxicity, and may decrease the rate of later brain metastases. Therefore, several cooperative groups and academic centers have initiated trials investigating lapatinib in the treatment of early-stage ErbB2 (HER2)-overexpressing breast cancer.

\section{Lapatinib in the Adjuvant Setting}

Based on the drug's effectiveness in advanced breast cancer, the role of lapatinib in the adjuvant setting for the treatment of ErbB2 (HER2)-positive breast cancer is currently being investigated. The dual tyrosine kinase (TK) inhibitor seems to be the ideal candidate for the treatment of early ErbB2-positive breast cancer for various reasons: i) lapatinib offers the unique convenience of oral use; ii) in contrast to trastuzumab, which prevents binding of growth factors at the extracellular ErbB2 domain, lapatinib acts by inhibiting the intracellular TKs of ErbB2 and ErbB1; iii) lapatinib has demonstrated ac-

\section{Schlüsselwörter}

Lapatinib - ErbB2-positives Mammakarzinom .

Adjuvant · Neoadjuvant · ALTTO-Studie - TEACH-Studie

\section{Zusammenfassung}

Lapatinib ist potenziell eine ideale Therapie im adjuvanten und neoadjuvanten Setting. Das liegt an der praktischen Handhabung (oral verfügbar, tägliche Einmalgabe), zudem hat die Substanz sowohl bei Patientinnen mit metastasiertem Mammakarzinom in der First-LineTherapie als auch in späteren Therapielinien seine antitumorale Aktivität gezeigt. Darüber hinaus ist der duale Tyrosinkinasehemmer mit einer nur geringen Kardiotoxizität assoziiert und hat das Potenzial, spätere Hirnmetastasen zu reduzieren. Vor diesem Hintergrund wurden von verschiedenen Studiengruppen und akademischen Zentren Studien mit Lapatinib bei Patientinnen mit ErbB2 (HER2)-positivem, frühen Mammakarzinom initiiert.

tivity in tumor xenografts of p95-positive BT474 breast cancer cells that are characterized by a truncated version of ErbB2 and to which trastuzumab cannot bind [1]; iv) lapatinib has demonstrated efficacy in trastuzumab-pretreated and resistant patients [2,3], and has shown promising results in patients with central nervous system (CNS) disease [4].

The Adjuvant Lapatinib and/or Trastuzumab Treatment Optimization (ALTTO) study is a 4-arm, open label, randomized, multicentre phase III trial evaluating lapatinib (arm A), trastuzumab (arm B), trastuzumab followed by lapatinib ( $\operatorname{arm} \mathrm{C})$, or concurrent treatment with both agents (arm D) for early stage ErbB2-positive breast cancer. Initially, 2 trial

\section{KARGER}

Fax +497614520714

Information@Karger.de

www.karger.com
(C) 2010 S. Karger GmbH, Freiburg
Prof. Dr. Christian Jackisch

Klinikum Offenbach

Frauenklinik

Starkenburgring 66, 63069 Offenbach, Germany

Tel. +49 69 8405-3850, Fax -4456

christian.jackisch@klinikum-offenbach.de 


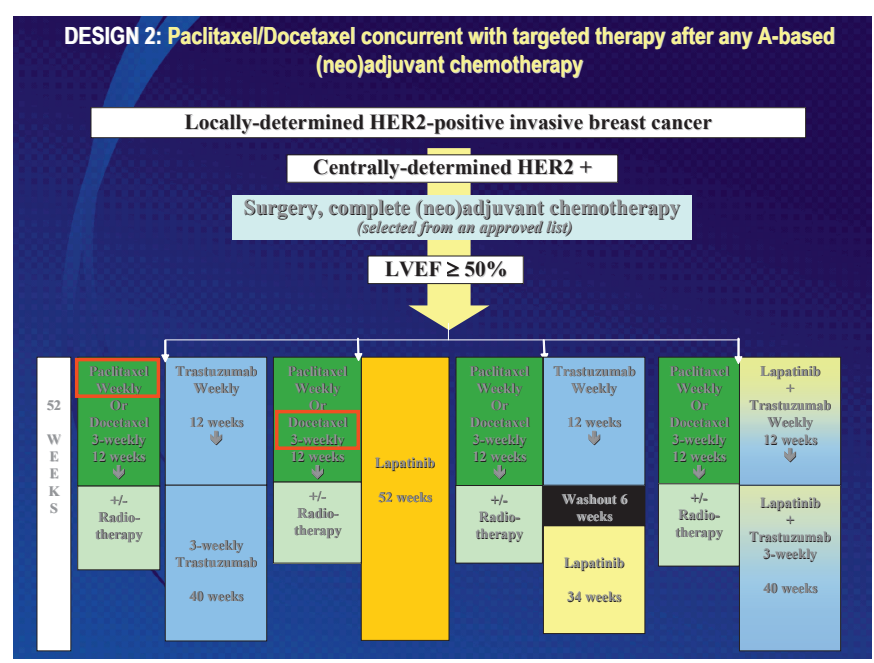

Fig. 1. Design 2 of the ALTTO trial.

designs were available: In design 1 , which is analogous to the design of the HERA trial, patients are randomized to one of the 4 anti-ErbB2 treatment strategies described above after adjuvant anthracycline-containing chemotherapy. In design 2 (fig. 1), the anti-ErbB2 therapy will commence concurrently with paclitaxel or docetaxel after surgery and anthracyclinebased chemotherapy. In the most recently implemented design 2B, patients receive an anthracycline-free chemotherapy regimen containing docetaxel and carboplatin. Chemotherapy is administered along with the anti-ErbB2 therapy. Design 2B will be implemented in the United States only. Patients will receive study treatment for 1 year (52 weeks), and will be followed up for a total of 10 years. The great variability of possible chemotherapeutic regimens reflects the fact that ALTTO, as a globally recruiting study, has to account for different treatment patterns in the participating countries. Patients included in ALTTO must have early-stage breast cancer. ErbB2 overexpression (immunohistochemistry (IHC) 3+ or fluorescence in situ hybridization (FISH) +) is confirmed by pathology testing. The ALTTO study will enroll 8,400 participants at about 1,300 sites in approximately 50 countries on 6 continents. The first patients were enrolled into the trial in June 2007; the study is projected to complete enrollment in 2010.

Currently, there is a therapeutic gap for many women with a diagnosis of early ErbB2-positive disease, whose adjuvant chemotherapy has been completed some time ago without having received trastuzumab (since the antibody was not available at that time). The randomized phase III study TEACH (Tykerb Evaluation After Chemotherapy) was designed to test lapatinib's efficacy in patients with ErbB2-positive early breast cancer. Eligible women must have completed adjuvant chemotherapy, be free of disease, and have either a new diagnosis and be unable or unwilling to receive trastuzumab or have a remote diagnosis of ErbB2-overexpressing

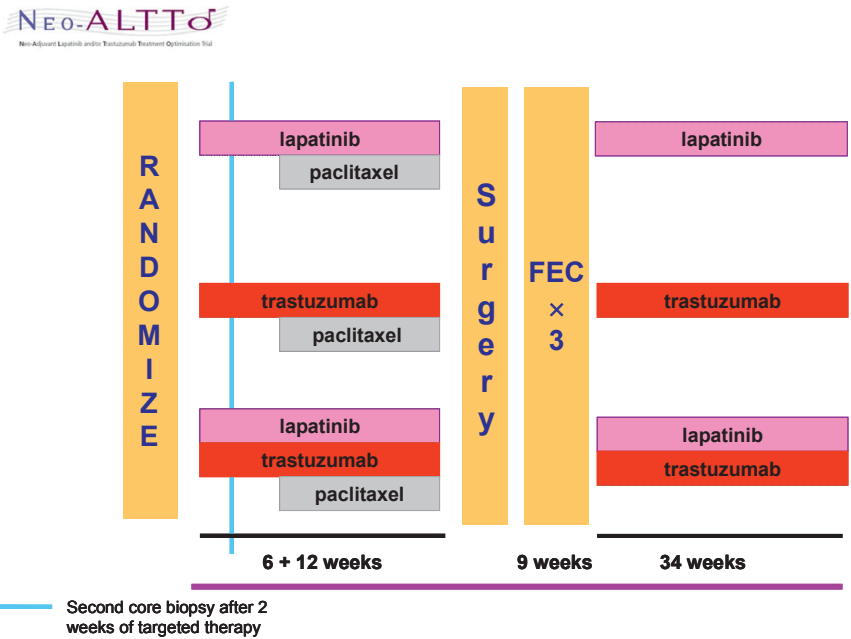

Fig. 2. Design of the Neo-ALTTO trial.

breast cancer and not have received prior trastuzumab. Approximately 3,000 women are enrolled from more than 450 centers in more than 30 countries. Participants were randomized to receive lapatinib $1,500 \mathrm{mg}$ or matching placebo orally administered once daily. Treatment is continued for a maximum of 12 months or until disease recurrence, development of a second primary cancer, withdrawal from study drug due to unacceptable toxicity, or consent withdrawal. All women will be followed up until death or until study closure. Disease-free survival was defined as the primary efficacy endpoint; study recruitment is completed.

\section{Lapatinib in the Neoadjuvant Setting}

Originally, neoadjuvant (primary systemic) therapy was standard of care for women with locally advanced and inflammatory breast cancers which are, by definition, inoperable. However, this approach is increasingly used for operable, early-stage disease as well, offering several advantages [5]: i) the use of neoadjuvant therapy increases the rate of breastconserving surgery; ii) given that a pathologic complete response (pCR) is a surrogate marker for improved clinical outcome, neoadjuvant therapy yields an opportunity to undertake correlative research by allowing in vivo assessment of tumor response to particular drug regimens; iii) the addition of trastuzumab to neoadjuvant chemotherapy has shown to significantly increase pCR, breast conservation rates, and event-free survival in women with primary operable, ErbB2overexpressing disease [6-8].

These promising results paved the way for integrating lapatinib in current neoadjuvant studies. The randomized, open label, multicenter phase III study Neo-ALTTO (Neoadjuvant Lapatinib and/or Trastuzumab Treatment Optimisation) was designed to compare the efficacy of neoadjuvant lapatinib, 


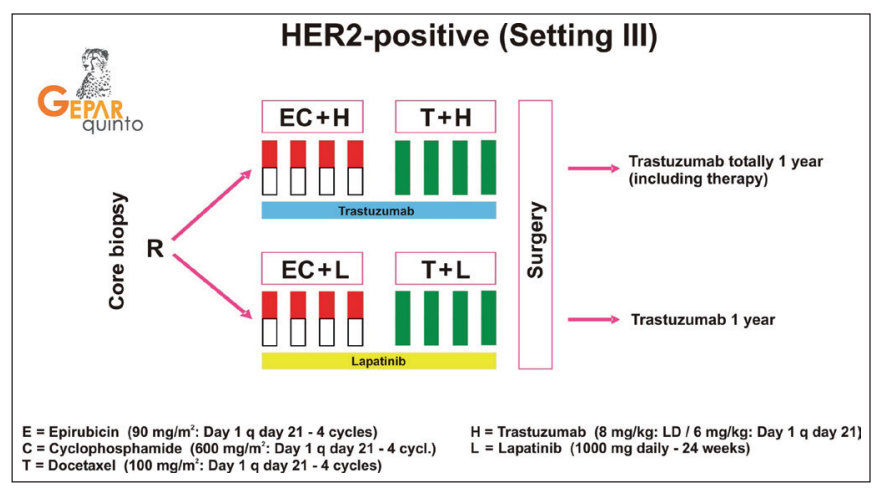

Fig. 3. Design of the Geparquinto trial: design of the ErbB2-positive cohort (Setting III). R: Randomization.

trastuzumab, or their combination, in combination with paclitaxel. The study is organized by BIG (Breast International Group) and the Spanish-based SOLTI (SOLid Tumour Intensification) Group. Patients are randomized to receive either lapatinib $1,500 \mathrm{mg}$ daily; trastuzumab $4 \mathrm{mg} / \mathrm{kg}$ intravenous (IV) load followed by $2 \mathrm{mg} / \mathrm{kg}$ IV weekly, or lapatinib $1,000 \mathrm{mg}$ daily with trastuzumab $4 \mathrm{mg} / \mathrm{kg}$ IV load followed by $2 \mathrm{mg} / \mathrm{kg}$ IV weekly for a total of 6 weeks (fig. 2). After this 'biological window', all patients continue on the same targeted therapy plus weekly paclitaxel $80 \mathrm{mg} / \mathrm{m}^{2}$ for a further 12 weeks, up to definitive surgery. After surgery, patients receive 3 courses of adjuvant chemotherapy with 5-fluorouracil, epirubicine, and cyclophosphamide (FEC), followed by the same targeted therapy that was begun in the neoadjuvant setting for a further 34 weeks (i.e. trastuzumab every 3 weeks, lapatinib, or combination). Patients receive study treatments for a total of 1 year (18 weeks pre-surgery, and 34 weeks post-surgery). The primary endpoint was defined as $\mathrm{pCR}$ rate at the time of surgery (time frame: 20-22 weeks). A total number of 450 women with ErbB2-positive breast cancer at about 130 sites in approximately 30 countries have been recruited. The study finished enrollment in November 2009.

To improve the individualization of therapy according to the tumors' sensitivity to chemotherapy as well as implementing small molecules with specific mechanism of action, the prospective, randomized, open label, multicenter phase III GeparQuinto trial has been initiated by the German Breast Group (GBG). In GeparQuinto, study patients will be allocated according to the ErbB2 status of the tumor as well as the sonographic response after the first 4 cycles of chemotherapy. Patients with ErbB2-positive breast cancer will receive either lapatinib or trastuzumab or lapatinib in addition to chemotherapy (fig. 3). Further experimental arms will include bevacizumab, an antiangiogenetic agent, and everolimus (RAD001), an mTOR inhibitor. Primary endpoint is the pCR rate of breast and lymph nodes. Enrolment started in November 2007 and will go on until fall 2010 with a planned accrual of 2,547 women.

\section{Disclosure Statement}

The authors were speakers and contributors to the meeting 'ErbB2 (HER2)-positives Mammakarzinom; 2. Münchner Brustkrebs-Symposium Update 2009', 23/24 October 2009 in Munich, sponsored by GlaxoSmithKline.

\section{References}

1 Xia W, Liu LH, Ho P, Spector NL: Truncated ErbB2 receptor (p95ErbB2) is regulated by heregulin through heterodimer formation with ErbB3 yet remains sensitive to the dual EGFR/ErbB2 kinase inhibitor GW572016. Oncogene 2004;23: 646-653.

2 Burstein H, Storniolo AM, Franco S, et al.: A phase II, open-label, multi-center study of lapatinib in two cohorts of patients with advanced or metastatic breast cancer who have progressed while receiving trastuzumab-containing regimens. Ann Oncol 2004;15(suppl 3):iii27.

3 Blackwell, KL, Kaplan EH, Franco SX, et al: A phase II, open-label, multi-center study of GW572016 in patients with trastuzumab-refractory metastatic breast cancer. J Clin Oncol 2004;22:3006.
4 Lin NU, Dieras V, Paul D, et al.: A phase II study of lapatinib for brain metastases in subjects with ErbB2-positive breast cancer following trastuzumab-based systemic therapy and cranial radiotherapy. Proc Am Soc Clin Oncol 2007;27(suppl):abstr 1012.

5 Lemieux J, Clemons M, Provencher L, et al.: The role of neoadjuvant HER2-targeted therapies in HER2-overexpressing breast cancers. Curr Oncol 2009;16:48-57.

-6 Buzdar AU, Ibrahim NK, Francis D, et al.: Significantly higher pathologic complete remission rate after neoadjuvant therapy with trastuzumab, paclitaxel, and epirubicin chemotherapy: results of a randomized trial in human epidermal growth factor receptor 2-positive operable breast cancer. J Clin Oncol 2005;23:3676-3685.
7 Coudert BP, Arnould L, Moreau L, et al.: Preoperative systemic (neo-adjuvant) therapy with trastuzumab and docetaxel for HER2-overexpressing stage II or III breast cancer: results of a multicenter phase II trial. Ann Oncol 2006;17:409-414.

8 Van Pelt AE, Mohsin S, Elledge RM, et al.: Neoadjuvant trastuzumab and docetaxel in breast cancer: preliminary results. Clin Breast Cancer 2003; 4:348-353. 